\title{
Extremely Broadband Stochastic Resonance of Light and Enhanced Energy Harvesting Enabled by Memory Effects in the Nonlinear Response
}

\author{
K. J. H. Peters $\odot,{ }^{1}$ Z. Geng $\odot,{ }^{1}$ K. Malmir $\odot,{ }^{2}$ J. M. Smith, ${ }^{2}$ and S. R. K. Rodriguez $\oplus^{1, *}$ \\ ${ }^{1}$ Center for Nanophotonics, AMOLF, Science Park 104, 1098 XG Amsterdam, Netherlands \\ ${ }^{2}$ Department of Materials, University of Oxford, Parks Road, Oxford OX1 3PH, United Kingdom
}

(Received 26 August 2020; accepted 4 May 2021; published 27 May 2021)

\begin{abstract}
We report the first observation of non-Markovian stochastic resonance (SR), and we discover that memory effects in the nonlinearity extremely enlarge the SR bandwidth. Our experimental system is an oilfilled microcavity which, driven by a continuous wave laser, has memory in its nonlinear optical response. Modulating the cavity length while adding noise to the driving laser, we observe a peak in the transmitted signal-to-noise ratio as a function of the noise variance. Through simulations, we reproduce our observations and extrapolate that the SR bandwidth could be 3000 times larger in our cavity than in a Kerr-nonlinear cavity. Experiments evidencing this memory-enhanced bandwidth across two decades are presented. As an extension of our results, we numerically demonstrate an order-of-magnitude enhancement in energy harvesting thanks to a nonlinearity with memory.
\end{abstract}

DOI: 10.1103/PhysRevLett.126.213901

Stochastic resonance $(\mathrm{SR})$ is a phenomenon wherein an optimum amount of noise amplifies the response of a nonlinear system to a periodic signal [1]. The essence of SR can be recognized in the behavior of a Brownian particle in a double well potential (DWP). A Brownian particle escapes from a potential well in a characteristic time $\tau_{\text {esc }} \propto \exp \left(E / k_{B} T\right)$, with $E$ the energy barrier and $k_{B} T$ the energy of thermal fluctuations [2]. Modulating the potential with period $T_{\text {mod }}=2 \tau_{\text {esc }}$ synchronizes the particle's Brownian motion with the modulation, resulting in two barrier crossings per period-stochastic resonance.

SR was introduced as an explanation for recurrent ice ages $[3,4]$. Since then, SR has been extensively observed in physics [5-15], chemistry [16-19], biology [20-24], ecology [25], psychophysics [26-28], climate science [29], finance [30,31], and social science [32]. Applications of SR to imaging [33-35] and mechanical engineering [36,37] have also emerged. To date, all observations of SR have been described within the Markov approximation neglecting memory effects. While non-Markovian dynamics have been observed in various systems [38-45] and are expected to modify SR [46-48], non-Markovian SR has not been experimentally reported.

In this Letter, we report the first observation of nonMarkovian SR and discover an extremely enlarged bandwidth thanks to memory effects in the nonlinearity. We investigate an oil-filled optical microcavity with thermooptical nonlinearity. Unlike the class of non-Markovian systems introduced by Mori [49] and described by generalized Langevin equations [50], the memory of our cavity is unrelated to its dissipation and noise spectrum. We drive our cavity with a laser to which we add a controlled amount of Gaussian white noise. By periodically modulating the cavity length, we imprint a signal on the laser. SR is evidenced by a peak in the transmitted signal-to-noise ratio (SNR). Simulations accounting for the memory time of the nonlinearity reproduce our observations, and suggest that the SR bandwidth should be 3000 times larger in our oilfilled cavity than in a Kerr-nonlinear cavity. Experimental indications of this memory-enhanced bandwidth are presented. Finally, we numerically demonstrate how memory effects in the nonlinearity substantially increase energy harvesting using a mechanical oscillator.

Figure 1(a) illustrates our setup, comprising a tunable microcavity filled with macadamia oil. The cavity is made by a planar and a concave mirror. The planar mirror is a $60 \mathrm{~nm}$ thick silver layer on a glass substrate. The concave mirror ( $7 \mu \mathrm{m}$ diameter, $12 \mu \mathrm{m}$ radius of curvature) is made by milling a glass substrate with a focused ion beam [51], and subsequently coating it with a distributed Bragg reflector (DBR). The DBR has a peak reflectance of $99.9 \%$ at $530 \mathrm{~nm}$. We use piezoelectric actuators to align and position the concave mirror and to modulate the planar mirror. Thanks to the strong optical confinement provided by the concave micromirror, we effectively probe a single optical mode throughout the modulation range. Indeed, the nearest mode is $\sim 10$ linewidths away. The cavity is driven by a $532 \mathrm{~nm}$ single-mode continuous wave laser, which heats the oil and causes its refractive index to decrease. A similar intensity-dependent refractive index underlies the observation of SR in Kerr-nonlinear cavities at cryogenic temperatures [9]. Unlike those cavities, our oil-filled cavity has: (i) a strong nonlinearity at room temperature, and (ii) noninstantaneous thermal relaxation leading to nonMarkovian dynamics. Our excitation and collection objectives both have $10 \times$ magnification and a numerical aperture 


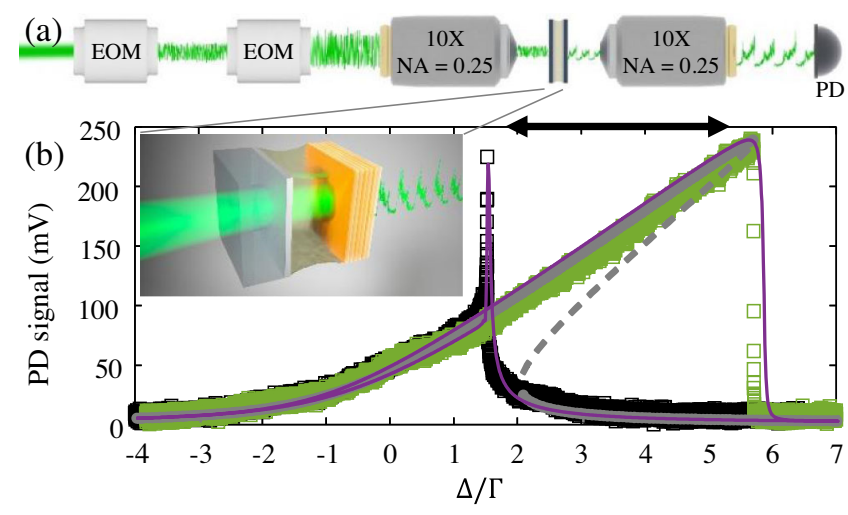

FIG. 1. (a) An oil-filled optical microcavity driven by a noisy laser. Electro-optic modulators (EOMs) add amplitude and phase noise to a continuous wave laser. The cavity length is controlled with a piezoelectric actuator, and the transmission is measured with a photodetector (PD). (b) Transmission, when slowly closing and opening the cavity, shown as black and green squares, respectively. The cavity length was converted to $\Delta / \Gamma$ [see Eq. (1)] using the laser frequency and the cavity resonance linewidth at low power. Solid and dashed gray curves are stable and unstable steady states, respectively. Purple curves are dynamic hysteresis simulations using Eq. (1) with $D=0$. Double-sided arrow indicates the $12 \mathrm{~nm}$ modulation range used in Fig. 2.

of 0.25 . In all experiments presented here, we drive the 15 th longitudinal and fundamental transverse cavity mode with a laser power of $7.8 \mathrm{~mW}$ at the excitation objective. The transmitted light is measured with a photodetector and an oscilloscope.

In a frame rotating at the laser frequency $\omega$, the light field $\alpha$ in our single-mode oil-filled cavity satisfies the following stochastic integrodifferential equation:

$$
\begin{aligned}
i \dot{\alpha}(t)= & {\left[-\Delta-i \frac{\Gamma}{2}+U \int_{0}^{t} d s K(t-s)\left[|\alpha(s)|^{2}-1\right]\right] \alpha(t) } \\
& +i \sqrt{\kappa_{L}} F+\frac{D}{\sqrt{2}}\left[\xi_{1}(t)+i \xi_{2}(t)\right] .
\end{aligned}
$$

$\Delta=\omega-\omega_{0}$ is the laser-cavity detuning, with $\omega_{0}$ the resonance frequency. $\Gamma=\gamma_{a}+\kappa_{L}+\kappa_{R}$ is the total loss rate, with $\gamma_{a}$ the absorption rate and $\kappa_{L, R}$ the input-output rates through the left and right mirror. $U$ is the nonlinearity strength, with noninstantaneous character captured by the time integral in Eq. (1). The integral contains the memory kernel $K(t)=\exp (-t / \tau) / \tau$ with $\tau$ the thermal relaxation time. This form of the kernel ensures that steady states $[\dot{\alpha}=0$ in Eq. (1)] are the same as for an instantaneous nonlinearity [52]. $F$ is the laser amplitude. $\xi_{1}(t)$ and $\xi_{2}(t)$ provide Gaussian white noise in the laser amplitude and phase, with combined variance $D^{2}$. For $\tau \rightarrow 0$ and $D=\sqrt{\Gamma / 2}$, Eq. (1) describes a Kerr-nonlinear cavity influenced by quantum fluctuations within the so-called truncated Wigner approximation [53]. Details about our calculations based on the xSPDE MATLAB toolbox [54], and values of model parameters are in the Supplemental Material [55].

For vanishing noise $(D=0)$ and large $F$ making the interaction energy larger than the losses (i.e., $U|\alpha|^{2} \gtrsim \Gamma$ ), Eq. (1) predicts optical bistability: two steady states with different intensity $|\alpha|^{2}$ at a single driving condition. To observe bistability, we measure the transmitted laser power at constant $F$ while opening and closing the cavity. The cavity length maps to $\Delta / \Gamma$, with $\Gamma$ the linewidth at low power. Figure 1(b) shows transmission measurements displaying bistability for $2 \lesssim \Delta / \Gamma \lesssim 5.5$. The overshoot at $\Delta / \Gamma \sim 1.5$ is due to the oil's noninstantaneous thermal relaxation [52]. Steady-state calculations assuming instantaneous nonlinearity [gray curves in Fig. 1(b)] do not reproduce this overshoot. In contrast, dynamic simulations [purple curves in Fig. 1(b)] based on Eq. (1) reproduce all our observations, including the overshoot. The overshoot width is determined by $T_{\text {mod }} / \tau$, with $\tau=10 \pm 1 \mu \mathrm{s}$ in our cavity (see Supplemental Material [55]).

For $D \neq 0$ and constant $(F, \Delta / \Gamma)$ yielding bistability, $\alpha$ randomly switches between states at a rate $\tau_{\text {esc }}^{-1} \propto \exp (D)$. This switching behavior is reminiscent of a Brownian particle in a DWP [56], albeit the analogy is not exact because a conservative potential cannot be defined for our driven-dissipative cavity. In the Supplemental Material [55], we show this switching behavior in stochastic trajectories of $|\alpha|^{2}$ and the corresponding probability density functions.

We demonstrate SR by modulating the cavity length and measuring the transmission. The cavity acts as a transducer, converting a mirror displacement to an optical signal. We set a subthreshold modulation amplitude of $12 \mathrm{~nm}$ [doublesided arrow in Fig. 1(b)], which is insufficient for deterministic switching given our laser power. Using the setup illustrated in Fig. 1(a), we add uncorrelated amplitude and phase noise by passing the laser through electro-optic modulators connected to different waveform generators. The noise power is approximately constant up to $200 \mathrm{kHz}$ (see Supplemental Material [55]), well above our cavity modulation frequency $f_{\text {mod }}=42.5 \mathrm{~Hz}$ and above the thermal relaxation rate $\tau^{-1}=100 \mathrm{kHz}$. Effectively, this corresponds to white noise. Moreover, the standard deviation of the noise $D$ is proportional to the peak-topeak (PP) voltage $V_{\mathrm{pp}}$ in the waveform generators [55].

Figures 2(a)-2(d) and 2(e)-2(h) show the experimental transmission and power spectral density (PSD), respectively, for different $V_{\mathrm{pp}}$. For the smallest $V_{\mathrm{pp}}$, the transmission is modulated by the changing cavity length [Fig. 2(a)] and the PSD shows a peak at $f_{\text {mod }}$ [Fig. 2(e)]. Noise in these measurements is due to the laser and detection and plays a minor role in our observations. Increasing $V_{\mathrm{pp}}$ induces random switches between high and low transmission states [Fig. 2(b)], which then maximally synchronize with the modulation when $V_{\mathrm{pp}}=70 \mathrm{~V}$ 

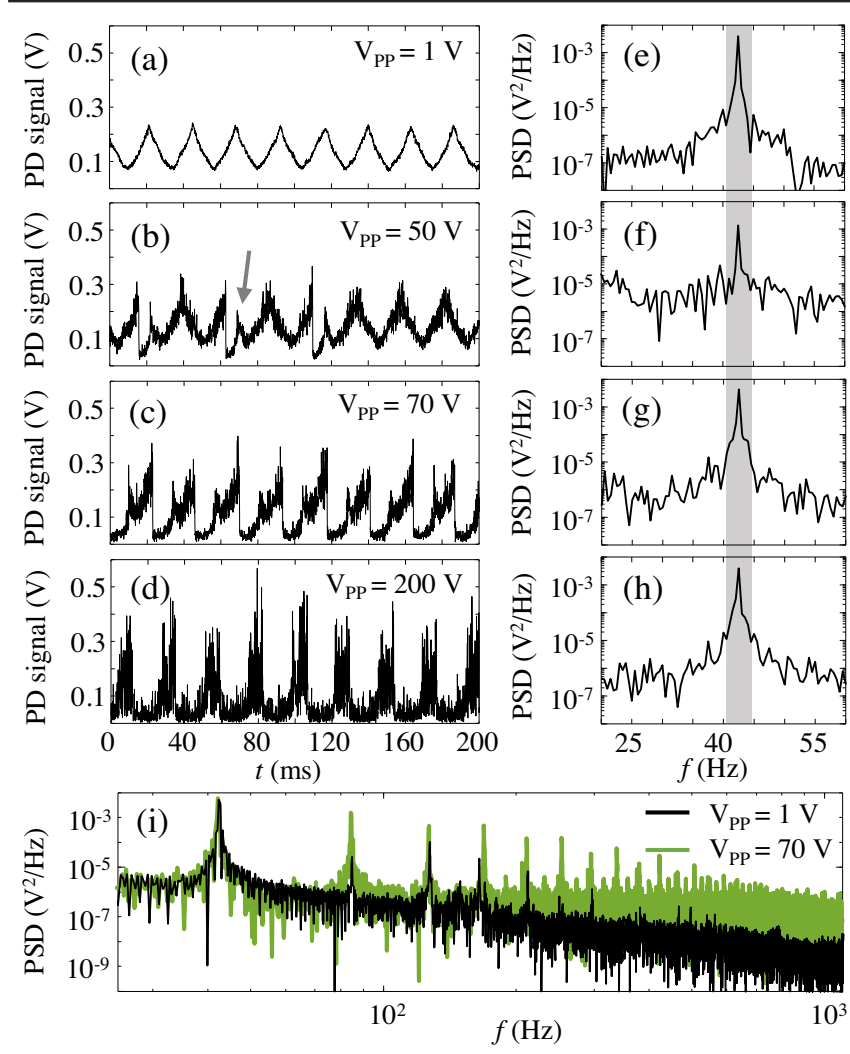

FIG. 2. (a)-(d) Transmission measurements as a function of time for increasing peak-to-peak (PP) voltage $V_{\text {pp }}$ supplied to the modulators adding noise. The arrow in (b) indicates the overshoot after a switching event. (e)-(h) Power spectral densities obtained by Fourier transforming the corresponding time traces in (a)-(d). Shaded area indicates the bandwidth of the fundamental. (i) Same data as in (e),(g), for an extended frequency range.

[Fig. 2(c)]. Figure 2(i) shows how this noise-assisted synchronization results in greater signal power, especially in the higher harmonics, while the output noise floor also increases. Further increasing $V_{\text {pp }}$ raises the noise floor and decreases power in higher harmonics (see Supplemental Material [55]), thereby resulting in an optimum noise strength for signal amplification. Note that we biased the system to the high-transmission state in our low-noise measurements [Figs. 2(a) and 2(b)]. Biasing the system to the low-transmission state would have resulted in a weaker signal for small $V_{\mathrm{pp}}$ and greater amplification.

Next, we analyze the transmitted signal-to-noise ratio. We define the SNR as the power in the first six harmonics including the fundamental $f_{\text {mod }}$ (integrated over a fixed $0.1 f_{\text {mod }}$ bandwidth), relative to the noise power. Figure 3(a) shows the SNR versus $V_{\mathrm{pp}}$. We distinguish three regimes. For small $V_{\mathrm{pp}}$, the SNR decreases with increasing $V_{\mathrm{pp}}$. This is due to more frequent random switching and a growing noise floor. For intermediate $V_{\mathrm{pp}}$, the SNR increases with $V_{\mathrm{pp}}$. Here, switching events increasingly synchronize with the modulation. Finally, for large $V_{\mathrm{pp}}$ the SNR decreases with $V_{\mathrm{pp}}$. This is evidenced in the reduced amplitude of the
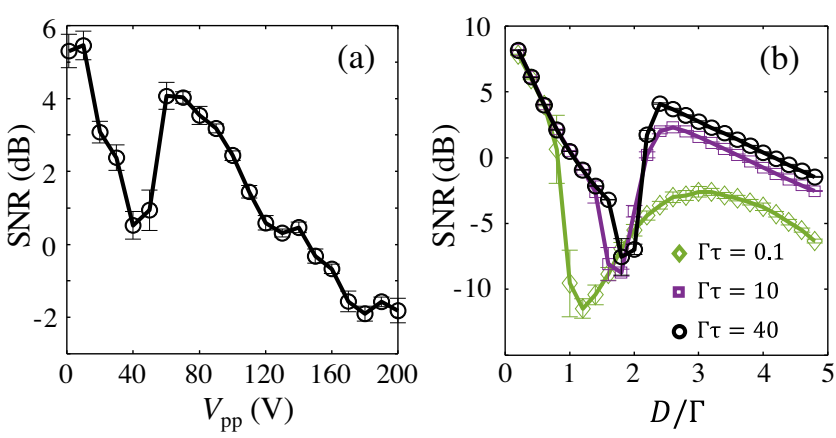

FIG. 3. (a) Measured signal-to-noise ratio (SNR) as a function of the peak-to-peak voltage $V_{\mathrm{pp}}$ supplied to the modulators adding noise. Experimental conditions are as in Fig. 2. Each data point is an average of ten measurements of two seconds ( $~ 800$ modulation cycles in total). (b) Calculated SNR as a function of the standard deviation $D$ of the noise (divided by the total loss rate $\Gamma$ ) for three values of the thermal relaxation time $\tau$. The ratio $T_{\text {mod }} / \tau=10^{3}$ is kept constant. Model parameters (see Supplemental Material [55]): $F / F_{c}=2.73, \Delta_{\min }=2.08 \Gamma$, $\Delta_{\max }=5.58 \Gamma$. Error bars indicate 1 standard deviation.

signal harmonics (see Supplemental Material [55]). In combination, these three regimes result in a SR peak around $V_{\mathrm{pp}}=70 \mathrm{~V}$. While not shown here, the height and width of the SR peak depend on the signal waveform [57].

We further validate our model by reproducing our SNR measurements. This will enable us to confidently simulate the effect of the memory time $\tau$ on SR over an extended range, which is not easily done in experiments. Figure 3(b) shows simulation results corresponding to the measurements in Fig. 3(a). We consider three values of $\tau$, keeping the ratio $T_{\text {mod }} / \tau$ constant since it determines the detuning range for which bistability occurs [52]. For $\tau>\Gamma^{-1}$, we observe a SNR peak growing with $\tau$ and remaining at approximately constant $D / \Gamma$. This behavior is due to two competing effects. On one hand, increasing $T_{\text {mod }}$ makes the SNR peak grow and shift to smaller $D$; the same occurs in standard Markovian SR. On the other hand, increasing $\tau$ slightly reduces the SNR peak and shifts it to larger $D$ (see Supplemental Material [55]); this effect is unique to our non-Markovian system. We attribute the peak SNR reduction to the overshoot following each switching [see Fig. 2(b)], which makes the signal further deviate from a pure sinusoidal. The shift to larger $D$ is associated with nonexponential distributions of residence times in the metastable states of the cavity [52], which is the hallmark of non-Markovian dynamics. Essentially, thermal relaxation imposes a high-frequency cutoff for switching events at $\tau^{-1}$. This results in SR at larger noise variance than in the Markovian case.

In Fig. 4(a), we calculate the average number of switches per cycle $n_{\text {avg }}$ as a function of $T_{\bmod }$, for different $\tau$ and constant $D \neq 0$ [55]. Recall that SR corresponds to $n_{\text {avg }} \approx 2$. For $\tau=0$, the black circles in Fig. 4 show $n_{\text {avg }}$ 


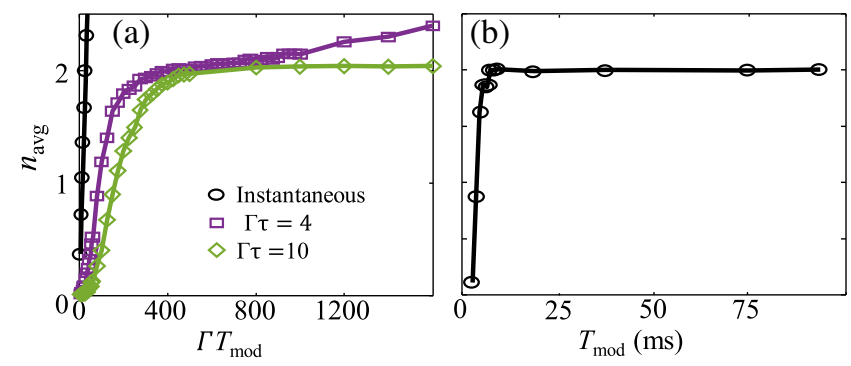

FIG. 4. (a) Calculated average number of switches per cycle $n_{\text {avg }}$ as a function of the modulation period $T_{\text {mod }}$ (times the total loss rate $\Gamma$ ), for fixed $D=5 \Gamma$. Model parameters are as in Fig. 3. (b) Measured $n_{\mathrm{avg}}$ as a function of $T_{\bmod }$ at $V_{\mathrm{pp}}=100 \mathrm{~V}$. The average is computed over 1200 modulation cycles. Error bars are smaller than data points.

simply increasing linearly with $T_{\text {mod }}$. In our system, this Markovian limit is reached for $\tau \ll \Gamma^{-1}$. More interesting behavior arises in the non-Markovian regime where $\tau>\Gamma^{-1}$. Taking $\Gamma \tau=4$ and $\Gamma \tau=10$ as examples, Fig. 4 shows a "plateau" at the SR condition $n_{\text {avg }} \approx 2$. This plateau represents an enlargement of the signal frequency range in which SR can be achieved, i.e., the SR bandwidth. Figure 4(b) shows similar behavior in our oil-filled cavity, where the plateau at $n_{\text {avg }} \approx 2$ evidences broadband SR.

The enlarged SR bandwidth is due to the slow nonlinearity of our system. At the $n_{\text {avg }} \approx 2$ plateau (one switch in the up ramp, one switch in the down ramp), an extra switch within the ramp requires much larger $T_{\text {mod }}$ or stronger noise. This is due to the long relaxation time to a steady state after a switch. The bandwidth is further enlarged by the slow increase of $n_{\text {avg }}$ with $T_{\text {mod }}$ at the edges of the plateau. Indeed, in the high-frequency regime where $n_{\text {avg }}<2$, bistability gradually disappears because the nonlinearity does not have time to build up [52]. For slow modulations, $n_{\text {avg }}$ increases linearly with $T_{\text {mod }}$ as in the Markovian case, albeit at a smaller rate. Consequently, SR becomes broadband. The SR bandwidth increases with $\tau$ as shown in the Supplemental Material [55]. By extrapolating the SR bandwidth up to our experimental $\tau=10 \mu \mathrm{s}$, and considering $\Gamma^{-1}=4 \mathrm{ps}$ in our experiments, we deduce that the SR bandwidth could be $3200 \pm 400$ times larger in our oil-filled cavity than in a Kerr-nonlinear cavity, if the fitted trend is maintained over a broad range. Because of the response time of our piezoelectric actuator and low-frequency mechanical fluctuations, we cannot perform measurements over such an extended range. Nevertheless, Fig. 4(b) clearly demonstrates broadband SR in experiments.

Inspired by the discovery of memory-enhanced SR bandwidth, we proceed to numerically investigate whether the same nonlinear memory can also improve the performance of a mechanical oscillator harvesting energy from noise. To this end, we consider the nonlinear energy harvester studied numerically by Cottone et al. [58] with two modifications. First, we endow the nonlinearity with memory. Second, for simplicity and generality, our energy harvester is driven by Gaussian white noise $\xi(t)$ instead of correlated noise. Our energy harvester has mass $m$, loss rate $\gamma$, and its displacement from equilibrium $x$ satisfies

$$
m \ddot{x}(t)=\left(a+b \int_{0}^{t} d s K(t-s) x(s)^{2}\right) x(t)-\gamma \dot{x}(t)+D \xi(t) .
$$

The constants $a$ and $b$ define a DWP $V(x)=-a x^{2} / 2+$ $b x^{4} / 4$ in the limit $\tau \rightarrow 0 . D^{2}$ is the noise variance. Calculation details are presented in the Supplemental Material [55].

Figure 5(a) shows the root-mean-square (rms) displacement $x_{\text {rms }}$ of our energy harvester with finite $\tau$, divided by the rms displacement $x_{\mathrm{rms}}^{0}$ of an energy harvester with $\tau=0$. Besides the change in $\tau$, the two systems have identical parameters. In the Markovian limit $\gamma \tau \ll 1$, $x_{\mathrm{rms}} / x_{\mathrm{rms}}^{0} \sim 1$ as expected. In the non-Markovian regime $\gamma \tau>1, \quad x_{\text {rms }}>x_{\text {rms }}^{0}$ demonstrates memory-enhanced energy harvesting. The enhancement is due to the existence of limit cycles with large-amplitude oscillations exceeding the distance between the minima of $V$ [55]. The increase in $x_{\mathrm{rms}} / x_{\mathrm{rms}}^{0}$ as $\tau \rightarrow \gamma^{-1}$ from above indicates that the limit cycles emerge from the interplay of different timescales $(\tau$ and $\gamma^{-1}$ ). Since somewhat similar limit cycles have been observed in nonlinear oscillators with constant time delay in their linear response [59], we analyze the relevance of our noninstantaneous nonlinearity for different $V$.

Figure 5(b) shows $x_{\mathrm{rms}}$ as a function of $a . a<0$ gives a single-well potential, while $a>0$ gives a DWP with barrier height growing with $a$ [see Fig. 5(b) inset]. For the Markovian case, $x_{\text {rms }}^{0}$ first increases and then decreases with $a$ [58]. $x_{\mathrm{rms}}^{0}$ decreases for large $a$ because the growing potential barrier traps the dynamics to a single well within
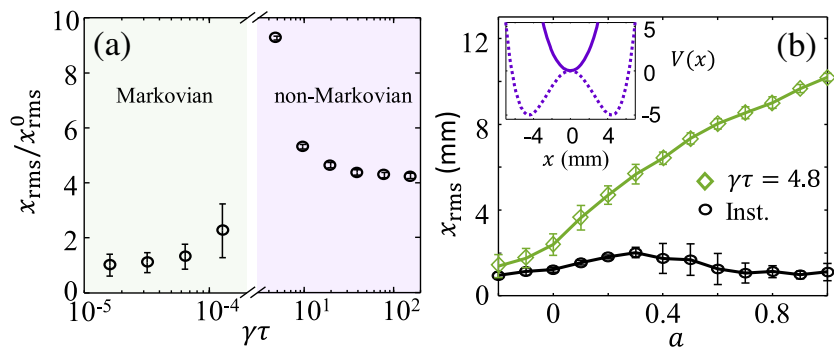

FIG. 5. (a) Enhanced rms displacement of an energy harvester with noninstantaneous nonlinearity relative to the instantaneous case, for variable memory time $\tau$. (b) rms displacement versus $a$ for the instantaneous case and for $\gamma \tau=4$.8. Inset: potential $V(x)$ for $a=-1$ (solid curve) and $a=1$ (dashed curve). Simulation parameters: $\gamma=0.016 \mathrm{~Hz}, \quad m=15.5 \mathrm{~kg}, \quad a=1, \quad b=0.05$, $D / \gamma=6.25$. Error bars indicate 1 standard deviation. 
the fixed energy harvesting time. Therefore, effective nonlinear energy harvesting is restricted to a narrow range of potentials in the Markovian regime. In contrast, the aforementioned limit cycles in the non-Markovian regime preclude the trapping of the dynamics to a potential well. This leads to a memory-enhanced energy harvesting for any potential, and much greater enhancement for a DWP. In particular, for $a=1$ (within the range considered in Ref. [58]), we observe an order-of-magnitude increase in the amount of energy harvested.

In summary, we demonstrated how memory effects in the nonlinearity render SR broadband. This result provides a plausible explanation for the occurrence of various noiseassisted processes at different frequencies within a single noisy environment, as observed in nature. Indeed, no natural system has a strictly instantaneous response. Future work could investigate the effects of colored or non-Gaussian noise, which enhance Markovian SR in certain regimes [60-62]. Furthermore, we predicted that memory effects in the nonlinearity can substantially improve energy harvesting. This exciting prediction, with potentially far-reaching technological implications, awaits experimental confirmation. Beyond single-resonator physics, our Letter paves the way for studying various noise-assisted processes (e.g., noise-assisted transport [6366]) in nonlinear and non-Markovian cavity arrays. Such arrays could be used to explore how memory effects can render complex networks resilient against breakdown of synchronization [67].

This work is part of the research programme of the Netherlands Organisation for Scientific Research (NWO). We thank Aurelien Trichet for help with sample fabrication, Pieter Rein ten Wolde, Femius Koenderink, Ewold Verhagen, Allard Mosk, and Sanli Faez for stimulating discussions, and Ricardo Struik and Niels Commandeur for technical support. S. R. K. R. acknowledges support from NWO Veni Grant No. 016.Veni.189.039.

*s.rodriguez@amolf.nl

[1] L. Gammaitoni, P. Hänggi, P. Jung, and F. Marchesoni, Stochastic resonance, Rev. Mod. Phys. 70, 223 (1998).

[2] H. Kramers, Brownian motion in a field of force and the diffusion model of chemical reactions, Physica (Amsterdam) 7, 284 (1940).

[3] R. Benzi, A. Sutera, and A. Vulpiani, The mechanism of stochastic resonance, J. Phys. A 14, L453 (1981).

[4] C. Nicolis, Long-term climatic transitions and stochastic resonance, J. Stat. Phys. 70, 3 (1993).

[5] S. Fauve and F. Heslot, Stochastic resonance in a bistable system, Phys. Lett. A 97, 5 (1983).

[6] B. McNamara, K. Wiesenfeld, and R. Roy, Observation of Stochastic Resonance in a Ring Laser, Phys. Rev. Lett. 60, 2626 (1988).

[7] A. Hibbs, A. Singsaas, E. Jacobs, A. Bulsara, J. Bekkedahl, and F. Moss, Stochastic resonance in a superconducting loop with a Josephson junction, J. Appl. Phys. 77, 2582 (1995).

[8] I. Y. Lee, X. Lia, B. Kosko, and C. Zhou, Nanosignal processing: Stochastic resonance in carbon nanotubes that detect subthreshold signals, Nano Lett. 3, 1683 (2003).

[9] H. Abbaspour, S. Trebaol, F. Morier-Genoud, M. T. Portella-Oberli, and B. Deveaud, Stochastic Resonance in Collective Exciton-Polariton Excitations Inside a GaAs Microcavity, Phys. Rev. Lett. 113, 057401 (2014).

[10] H. Abbaspour, S. Trebaol, F. Morier-Genoud, M. T. Portella-Oberli, and B. Deveaud, Spinor stochastic resonance, Phys. Rev. B 91, 155307 (2015).

[11] W. J. Venstra, H. J. Westra, and H.S. Van Der Zant, Stochastic switching of cantilever motion, Nat. Commun. 4, 2624 (2013).

[12] F. Monifi, J. Zhang, S. Özdemir, B. Peng, Y. Liu, F. Bo, F. Nori, and L. Yang, Optomechanically induced stochastic resonance and chaos transfer between optical fields, Nat. Photonics 10, 399 (2016).

[13] F. Ricci, R. A. Rica, M. Spasenović, J. Gieseler, L. Rondin, L. Novotny, and R. Quidant, Optically levitated nanoparticle as a model system for stochastic bistable dynamics, Nat. Commun. 8, 15141 (2017).

[14] A. Chowdhury, S. Barbay, M. G. Clerc, I. Robert-Philip, and R. Braive, Phase Stochastic Resonance in a Forced Nanoelectromechanical Membrane, Phys. Rev. Lett. 119, 234101 (2017).

[15] T. Wagner, P. Talkner, J. C. Bayer, E. P. Rugeramigabo, P. Hänggi, and R. J. Haug, Quantum stochastic resonance in an ac-driven single-electron quantum dot, Nat. Phys. 15, 330 (2019).

[16] D. S. Leonard and L. E. Reichl, Stochastic resonance in a chemical reaction, Phys. Rev. E 49, 1734 (1994).

[17] A. Guderian, G. Dechert, K.-P. Zeyer, and F. Schneider, Stochastic resonance in chemistry. 1. The BelousovZhabotinsky reaction, J. Phys. Chem. 100, 4437 (1996).

[18] A. Förster, M. Merget, and F. Schneider, Stochastic resonance in chemistry. 2. The peroxidase-oxidase reaction, J. Phys. Chem. 100, 4442 (1996).

[19] W. Hohmann, J. Müller, and F. Schneider, Stochastic resonance in chemistry. 3. The minimal-bromate reaction, J. Phys. Chem. 100, 5388 (1996).

[20] J. K. Douglass, L. A. Wilkens, E. Pantazelou, and F. Moss, Noise enhancement of information transfer in crayfish mechanoreceptors by stochastic resonance, Nature (London) 365, 337 (1993).

[21] S. M. Bezrukov and I. Vodyanoy, Noise-induced enhancement of signal transduction across voltage-dependent ion channels, Nature (London) 378, 362 (1995).

[22] F. Jaramillo and K. Wiesenfeld, Mechanoelectrical transduction assisted by Brownian motion: A role for noise in the auditory system, Nat. Neurosci. 1, 384 (1998).

[23] D. F. Russell, L. A. Wilkens, and F. Moss, Use of behavioural stochastic resonance by paddle fish for feeding, Nature (London) 402, 291 (1999).

[24] T. Mori and S. Kai, Noise-Induced Entrainment and Stochastic Resonance in Human Brain Waves, Phys. Rev. Lett. 88, 218101 (2002).

[25] Blarer and Doebeli, Resonance effects and outbreaks in ecological time series, Ecol. Lett. 2, 167 (1999). 
[26] J. J. Collins, T. T. Imhoff, and P. Grigg, Noise-enhanced tactile sensation, Nature (London) 383, 770 (1996).

[27] E. Simonotto, M. Riani, C. Seife, M. Roberts, J. Twitty, and F. Moss, Visual Perception of Stochastic Resonance, Phys. Rev. Lett. 78, 1186 (1997).

[28] L. M. Ward, A. Neiman, and F. Moss, Stochastic resonance in psychophysics and in animal behavior, Biol. Cybern. 87, 91 (2002).

[29] A. Ganopolski and S. Rahmstorf, Abrupt Glacial Climate Changes Due to Stochastic Resonance, Phys. Rev. Lett. 88, 038501 (2002).

[30] M. Xiao-Ming, S. Kai, and O. Qi, Stochastic resonance in a financial model, Chin. Phys. 11, 1106 (2002).

[31] A. Krawiecki and J. Hołyst, Stochastic resonance as a model for financial market crashes and bubbles, Physica (Amsterdam) 317A, 597 (2003).

[32] R. Wallace, D. Wallace, and H. Andrews, AIDS, tuberculosis, violent crime, and low birthweight in eight US metropolitan areas: Public policy, stochastic resonance, and the regional diffusion of inner-city markers, Environ. Plan A 29, 525 (1997).

[33] R. J. Marks, B. Thompson, M. A. El-Sharkawi, W. L. J. Fox, and R. T. Miyamoto, Stochastic resonance of a threshold detector: Image visualization and explanation, in IEEE Symp. Circ. Syst., Vol. 4 (IEEE, New York, 2002), p. IV.

[34] V. S. Rallabandi, Enhancement of ultrasound images using stochastic resonance-based wavelet transform, Computerized Medical Imaging and Graphics 32, 316 (2008).

[35] D. V. Dylov and J. W. Fleischer, Nonlinear self-filtering of noisy images via dynamical stochastic resonance, Nat. Photonics 4, 323 (2010).

[36] H. Niaoqing, C. Min, and W. Xisen, The application of stochastic resonance theory for early detecting rub-impact fault of rotor system, Mech. Syst. Signal Pr. 17, 883 (2003).

[37] J. Li, X. Chen, and Z. He, Multi-stable stochastic resonance and its application research on mechanical fault diagnosis, J. Sound Vib. 332, 5999 (2013).

[38] P. Hanggi, T. J. Mroczkowski, F. Moss, and P. V. E. McClintock, Bistability driven by colored noise: Theory and experiment, Phys. Rev. A 32, 695 (1985).

[39] L. Liebovitch and J. Sullivan, Fractal analysis of a voltagedependent potassium channel from cultured mouse hippocampal neurons, Biophys. J. 52, 979 (1987).

[40] S. Mercik and K. Weron, Stochastic origins of the longrange correlations of ionic current fluctuations in membrane channels, Phys. Rev. E 63, 051910 (2001).

[41] J. Houlihan, D. Goulding, T. Busch, C. Masoller, and G. Huyet, Experimental Investigation of a Bistable System in the Presence of Noise and Delay, Phys. Rev. Lett. 92, 050601 (2004).

[42] B.-H. Liu, L. Li, Y.-F. Huang, C.-F. Li, G.-C. Guo, E.-M. Laine, H.-P. Breuer, and J. Piilo, Experimental control of the transition from Markovian to non-Markovian dynamics of open quantum systems, Nat. Phys. 7, 931 (2011).

[43] K. H. Madsen, S. Ates, T. Lund-Hansen, A. Löffler, S. Reitzenstein, A. Forchel, and P. Lodahl, Observation of nonMarkovian Dynamics of a Single Quantum Dot in a Micropillar Cavity, Phys. Rev. Lett. 106, 233601 (2011).

[44] U. Hoeppe, C. Wolff, J. Küchenmeister, J. Niegemann, M. Drescher, H. Benner, and K. Busch, Direct Observation of Non-Markovian Radiation Dynamics in 3D Bulk Photonic Crystals, Phys. Rev. Lett. 108, 043603 (2012).

[45] Y.-N. Lu, Y.-R. Zhang, G.-Q. Liu, F. Nori, H. Fan, and X.-Y. Pan, Observing Information Backflow from Controllable Non-Markovian Multichannels in Diamond, Phys. Rev. Lett. 124, 210502 (2020).

[46] A. Neiman and W. Sung, Memory effects on stochastic resonance, Phys. Lett. A 223, 341 (1996).

[47] I. Goychuk and P. Hänggi, Non-Markovian Stochastic Resonance, Phys. Rev. Lett. 91, 070601 (2003).

[48] T. Prager and L. Schimansky-Geier, Stochastic Resonance in a Non-Markovian Discrete State Model for Excitable Systems, Phys. Rev. Lett. 91, 230601 (2003).

[49] H. Mori, Transport, collective motion, and Brownian motion, Prog. Theor. Phys. 33, 423 (1965).

[50] P. Hänggi, Correlation functions and masterequations of generalized (non-Markovian) Langevin equations, Z. Phys. 31, 407 (1978).

[51] A. A. P. Trichet, P. R. Dolan, D. M. Coles, G. M. Hughes, and J.M. Smith, Topographic control of open-access microcavities at the nanometer scale, Opt. Express 23, 17205 (2015).

[52] Z. Geng, K. J. H. Peters, A. A. P. Trichet, K. Malmir, R. Kolkowski, J. M. Smith, and S. R. K. Rodriguez, Universal Scaling in the Dynamic Hysteresis, and Non-Markovian Dynamics, of a Tunable Optical Cavity, Phys. Rev. Lett. 124, 153603 (2020).

[53] I. Carusotto and C. Ciuti, Quantum fluids of light, Rev. Mod. Phys. 85, 299 (2013).

[54] S. Kiesewetter, R. Polkinghorne, B. Opanchuk, and P. D. Drummond, xSPDE: Extensible software for stochastic equations, SoftwareX 5, 12 (2016).

[55] See Supplemental Material at http://link.aps.org/ supplemental/10.1103/PhysRevLett.126.213901 for details about the calculations, the thermal relaxation time, the switching behavior in simulated trajectories, the input noise properties, the measured power spectral density for different noise variances, the method used for detecting switching events, the scaling of the stochastic resonance bandwidth with the memory time of the nonlinearity, and the energy harvester with noninstantaneous nonlinearity.

[56] H. Risken, C. Savage, F. Haake, and D. F. Walls, Quantum tunneling in dispersive optical bistability, Phys. Rev. A 35, 1729 (1987).

[57] V. Gandhimathi, K. Murali, and S. Rajasekar, Stochastic resonance with different periodic forces in overdamped two coupled anharmonic oscillators, Chaos, Solitons Fractals 30, 1034 (2006).

[58] F. Cottone, H. Vocca, and L. Gammaitoni, Nonlinear Energy Harvesting, Phys. Rev. Lett. 102, 080601 (2009).

[59] J. Cantisán, M. Coccolo, J. M. Seoane, and M. A. Sanjuán, Delay-induced resonance in the time-delayed Duffing oscillator, Int. J. Bifurcation Chaos Appl. Sci. Eng. 30, 2030007 (2020).

[60] D. Nozaki, D. J. Mar, P. Grigg, and J. J. Collins, Effects of Colored Noise on Stochastic Resonance in Sensory Neurons, Phys. Rev. Lett. 82, 2402 (1999).

[61] M. Fuentes, R. Toral, and H.S. Wio, Enhancement of stochastic resonance: The role of non Gaussian noises, Physica (Amsterdam) 295A, 114 (2001). 
[62] F. J. Castro, M. N. Kuperman, M. Fuentes, and H. S. Wio, Experimental evidence of stochastic resonance without tuning due to non-Gaussian noises, Phys. Rev. E 64, 051105 (2001).

[63] M. B. Plenio and S. F. Huelga, Dephasing-assisted transport: quantum networks and biomolecules, New J. Phys. 10, 113019 (2008).

[64] F. Caruso, A. W. Chin, A. Datta, S. F. Huelga, and M. B. Plenio, Highly efficient energy excitation transfer in lightharvesting complexes: The fundamental role of noiseassisted transport, J. Chem. Phys. 131, 105106 (2009).
[65] S. Viciani, M. Lima, M. Bellini, and F. Caruso, Observation of Noise-Assisted Transport in an All-Optical Cavity-Based Network, Phys. Rev. Lett. 115, 083601 (2015).

[66] G. R. Ramirez-San Juan, A. J. Mathijssen, M. He, L. Jan, W. Marshall, and M. Prakash, Multi-scale spatial heterogeneity enhances particle clearance in airway ciliary arrays, Nat. Phys. 16, 958 (2020).

[67] Z.-H. Lin, M. Feng, M. Tang, Z. Liu, C. Xu, P. M. Hui, and Y.-C. Lai, Non-Markovian recovery makes complex networks more resilient against large-scale failures, Nat. Commun. 11, 2490 (2020). 